\title{
EL PRINCIPIO DE SPALLANZANI
}

\section{THE SPALLANZANI PRINCIPLE}

\section{Emilio Ridruejo}

Universidad de Valladolid

\section{Resumen}

El italiano Lazzaro Spallanzani (1729 - 1799) es bien conocido como el científico que desmontó las teorías de la generación espontánea. Pero Spallanzani fue también un pionero en el estudio de la regeneración de los seres vivos. En 1768 publicó el Prodromo, una obra sobre reproducción animal, en el que se interesaba también por la regeneración. Spallanzani describió varios tipos de regeneración, la de la cola de la rana y de las extremidades de la salamandra. Antes, en una carta dirigida en 1766 al biólogo suizo Charles Bonnet había descrito también la regeneración de la lombriz, de la babosa o del caracol.

PALABRAS CLAVE: Spallanzani, principio, lenguaje

\begin{abstract}
The Italian Lazzaro Spallanzani (1729 - 1799) is well known as the scientist who dismantled theories of spontaneous generation. But Spallanzani was also a pioneer in the study of the regeneration of living beings. In 1768 he published the Prodromo, a work on animal reproduction, in which he was also interested in regeneration. Spallanzani described several types of regeneration, that of the frog's tail and the tips of the salamander. Before, in a letter addressed in 1766 to the Swiss biologist Charles Bonnet, he had also described the regeneration of the earthworm, the slug or the snail.
\end{abstract}

KEY WORDS: Spallanzani, principle, language 
El italiano Lazzaro Spallanzani $(1729$ - 1799) es bien conocido como el científico que desmontó las teorías de la generación espontánea. Pero Spallanzani fue también un pionero en el estudio de la regeneración de los seres vivos. En 1768 publicó el Prodromo, una obra sobre reproducción animal, en el que se interesaba también por la regeneración. Spallanzani describió varios tipos de regeneración, la de la cola de la rana y de las extremidades de la salamandra. Antes, en una carta dirigida en 1766 al biólogo suizo Charles Bonnet había descrito también la regeneración de la lombriz, de la babosa o del caracol. Su correspondencia se refería a los problemas de la generación mediante gérmenes, que Bonnet había supuesto en los casos de regeneración. Spallanzani formuló un principio según el cual los tejidos regenerados no son iguales a los preexistentes y, además, que la regeneración tiene lugar con más facilidad cuanto más joven es el ejemplar afectado. De hecho, se ha descrito que incluso en mamíferos muy jóvenes puede tener lugar tal proceso: cuando se corta la falange terminal del dedo de las extremidades de ratones de hasta tres semanas regeneran una falange nueva, aunque más corta y con forma de gancho.

Las lenguas, como los seres vivos, experimentan procesos de cambio y regeneración. Dado que no todos los sistemas evolucionan con la misma facilidad ¿es posible proponer para las lenguas un principio similar al de Spallanzani? Sin aceptar en absoluto la teoría planteada en el siglo XIX por Schleicher de que las lenguas se comportan como organismos vivos, hay, sin embargo, aspectos de la hipótesis de Spallanzani que podrían coincidir con algunos procesos que tienen lugar en las lenguas naturales. Y es que, sorprendentemente, los cambios lingüísticos, al menos en algunos casos, también se ajustan a los parámetros de mayor frecuencia y facilidad vinculados a las entidades más jóvenes o más recientes.

\section{FACTORES QUE AFECTAN A LA VELOCIDAD DE LOS CAMBIOS}

No hay muchas investigaciones acerca de la velocidad y frecuencia de los cambios lingüísticos. Ciertamente se ha atestiguado la gran rapidez con la que tienen lugar las modificaciones que son precisas para configurar los criollos. $Y$ si algo hemos de entender como joven desde el punto de vista lingüístico, lo son los criollos. Surgen a partir de pidgins (o, al menos, a partir de lenguas en contacto muy íntimo, de koinés) y las estructuras de las lenguas de origen son modificadas con arreglo a determinados patrones de transparencia, orden y simplicidad. Aunque los criollistas tienen opiniones no coincidentes, algunos opinan que la criollización tiene lugar en solo una generación. Otros plantean periodos más largos. Pero, dado que los criollos hoy conocidos resultan de la evolución de pidgins producidos a partir de los grandes procesos de colonización de la Edad Moderna, su desarrollo, desde luego, ha sido muy rápido y está bien documentado. Pentland creía que los cambios del inglés al Wes-kon (en África occidental) fue el cambio lingüístico más rápido nunca conocido (Bakker 2000). El caso contrario, en el que también se aceleran exageradamente los cambios, es en las denominadas lenguas en atrición. No hay más que ver la fortísima degradación en un siglo del denominado español isleño de la Luisiana (Holloway 1997). 
En las lenguas históricas, las de evolución continuada durante un tiempo indefinido, entre los diferentes sistemas que las configuran, no todos cambian de la misma manera y a la misma velocidad. Aunque puede haber circunstancias muy diversas que las afectan, por ejemplo, el contacto con otras lenguas, no todos los componentes son reemplazados con la misma facilidad y a la misma velocidad. Es bien conocido que los rasgos suprasegmentales son duraderos y que la morfología y la sintaxis tienen también gran persistencia. En los sistemas fonológicos y gramaticales los cambios son lentos, dado que la modificación de un elemento implica alteraciones que afectan a todos los componentes del sistema. Por el contrario, el léxico es el ámbito en el que las innovaciones son más abundantes. Y dentro del léxico, el denominado cultural o técnico. Ya Sapir, quien estaba especialmente interesado en las relaciones culturales existentes entre diversos grupos de lenguas amerindias, había observado que los tecnicismos léxicos reflejan incorporaciones ocasionadas en un determinado momento cultural, bien como creaciones, bien como préstamos: el inglés spinster, 'soltera' (derivado de spin, 'rueca') solo pudo desarrollarse cuando se utilizaba la rueca (Sapir 1958, 434); bigote en español puede surgir solo cuando los que utilizaban ese aditamento capilar se caracterizaban por exclamar bei Got, es decir en un momento de contacto cultural con soldados alemanes.

\section{LAS VARIEDADES COLOQUIALES}

Otro aspecto que hay que tomar en consideración para el examen de la velocidad de los cambios en una lengua son sus variedades diafásicas. Es evidente que no presentan la misma estabilidad las variedades orales y espontáneas que las que se acomodan a una norma externa, la caracterizada por Havranek (1966 [1936]) como establecida por instituciones sociales y a través de sistemas de educación e información. La fijación normativa mediante recursos institucionales, hace que sea notablemente estable. La escritura, que le es inherente, como convención secundaria, puede ser muy insensible a la evolución $\mathrm{Y}$, además, la escritura implica una dependencia muy clara de variedades formales.

Al contrario, la lengua hablada en condiciones de espontaneidad, proximidad entre los interlocutores e informalidad temática, es decir, lo que habitualmente se entiende como variedad coloquial (Briz 1998; 40-44), presenta características diferentes. Obviamente, la variedad coloquial se ajusta a una norma interna, entendida en el sentido de Bloomfield (1964 [1927]:, 227). Se acomoda a los convenios por los cuales los hablantes toman como modelo los productos lingüísticos de algunos individuos de algún prestigio en la comunidad y procuran conformar sus relaciones lingüísticas de la forma más próxima posible a los modelos así establecidos. Ello afecta a todo tipo de componentes lingüísticos, desde los fónicos hasta los pragmáticos. Esa norma interna, por tanto, es sensible a las condiciones en que se emiten los mensajes, a factores como el tipo de acto de habla, la ironía, los contenidos temáticos, el número, la naturaleza y la relación entre los interlocutores. Sin embargo, la norma externa, la fijada institucionalmente, tiene claramente un peso menor que en las variedades no coloquiales. El discurso no espontáneo, esto es preparado, permite una mayor reflexión metalingüística que conduce, a no ser que se busque una alteración voluntaria (como sucede en ocasiones en la literatura cómica o costumbrista), al mayor respeto de la norma externa. Si no existe proximidad social entre los interlocutores, estos intentarán actuar en 
correspondencia con su estatus social, con su posición relativa de poder, y un componente básico para ello será el ajustar sus intercambios lingüísticos a lo especificado en la norma socialmente admitida y fijada. En cambio, la inmediatez y equiparación social de los interlocutores permite desentenderse de los aspectos más rígidos de la norma. Además, la proximidad conduce al empleo más frecuente de los recursos afectivos e intensificadores (Briz 1998, 135-140). De una parte, los que están codificados y que, consiguientemente, no suponen innovación alguna: el empleo de sufijos diminutivos o despectivos, los hipocorísticos, pero también otros novedosos, basados en la ironía, en la metáfora o la metonimia.

Por otra parte, en el intercambio formal suele darse una temática especializada, y ello obligará a elecciones léxicas también especializadas, es decir a tecnicismos, hayan sido fijados o no normativamente. En el coloquio, la variedad temática que suele ser habitual, puede afectar a la amplitud de las elecciones léxicas. En el coloquio también son abundantes las innovaciones que buscan la economía comunicativa: el empleo de apócopes o de suspensiones, aunque, claro está, que otros mecanismos similares, como el empleo de siglas, son más frecuentes en los tecnolectos formales.

Como consecuencia de todo lo anterior, al comparar las variedades coloquiales con las formales, escritas u orales, se puede advertir fácilmente que en las primeras, la aceptación de innovaciones es mayor. En el ámbito fónico, las variedades coloquiales, en virtud de las relaciones de proximidad entre los interlocutores, se caracterizan por la aceptación de rasgos que están estigmatizados en la conversación formal. En la gramática, la menor presión de la norma externa facilita los cambios de carácter analógico y los que se fundan en el principio de la mayor transparencia de las marcas. Desde el punto de vista de la pragmática, la proximidad entre los interlocutores, el conocimiento compartido del entorno y el contexto, así como una característica esencial de la variedad coloquial, la intensificación, todo sumado, facilita la resolución de ambigüedades y, por tanto, también los cambios semánticos basados en la ironia, la metáfora o la metonimia.

Si bien es más probable que las variedades coloquiales acepten con mayor facilidad las innovaciones, ¿tales innovaciones son persistentes o, al contrario, más efímeras que las introducidas en otras variedades distintas? Esto es ¿se cumple o no un principio semejante al de Spallanzani para los seres vivos?

\section{EL LÉXICO}

Si hay un ámbito en el que son más abundantes las innovaciones en las variedades coloquiales, este es el del léxico, que se configura entonces como especialmente apropiado para examinar las incorporaciones y el grado de persistencia de estas.

Con cierta frecuencia se han descrito las innovaciones léxicas que se producen en ámbitos marginales, en las jergas, y también entre los jóvenes, a la vez que se ha señalado la rapidez con se producen cambios. En estos casos, las innovaciones suelen proceder de las variedades coloquiales y aquí radica interés. Naturalmente el léxico coloquial no pertenece a ninguna franja de edad determinada, es utilizado por todos los individuos de una comunidad. Sin embargo, las innovaciones sí que se recogen 
dominantemente entre algunos grupos, especialmente caracterizados en cuanto a su edad, aunque también tal como ha sido estudiado en sociolingüística, sobre todo en ambientes socioculturales marcados como marginales (Penélope Eckert 1989).

Fernando Lázaro Carreter (1980) en los años ochenta, al describir las innovaciones que encontraba entre los jóvenes, señalaba que se trataba de componentes de un código que se imponía por parte de la comunidad a la que se incorporaban, de tal manera que el individuo protegido por sus peculiaridades, podía utilizarlo sin ninguna responsabilidad propia, y ésta quedaba transferida al grupo, que, a su vez resultaba reforzado en su entidad frente al resto de la sociedad y especialmente de la sociedad de las gentes de más edad. En estos hechos puede fundarse, probablemente, la inestabilidad del habla juvenil. El nacimiento y la desaparición de los grupos constituidos por los jóvenes son muy rápidos, precisamente porque su evolución se asocia con una franja de edad y nuevos grupos exigen una constante renovación de las marcas que los caracterizan, marcas, lingüísticas ciertamente, pero no más que otras extralingüísticas (en el vestido, en el peinado, en el tatuaje, en los pendientes, etc.) (Riviére 1989). La renovación de unas marcas, a su vez, trae consigo el rechazo de las anteriores, que ahora pasan a ser caracterizadoras de otros grupos de edad o, simplemente, caen en desuso.

Se ha supuesto que hay una notable semejanza entre las innovaciones del habla de los jóvenes y los que proceden de las jergas de militares o de delincuentes. Efectivamente, algunos items léxicos documentados inicialmente entre jóvenes, como hacer imaginarias o estar al loro, tienen ese origen, respectivamente militar y carcelario. Algunos de los recursos más empleados, truncamientos, deformaciones, extranjerismos son también propios del argot (Rodríguez Díez 1996). No obstante, Sanmartín (2006 [1998], 211) al comparar la lengua de los jóvenes con el argot de la delincuencia, encuentra que existen tres rasgos diferenciales entre ambas. En primer lugar, son distintos los ámbitos temáticos en los que se generan las innovaciones jergales, entre los delincuentes sólo tienen lugar en relación con asuntos específicos de su actividad (armas, delitos, drogas, etc.) mientras que entre los jóvenes el campo es mucho más general. En la jerga de los delincuentes se ha producido una hibridación con el caló que falta, en términos generales, en el habla de los jóvenes $y$, finalmente, entre los delincuentes el lenguaje jergal alcanza también a la comunicación no verbal, algo que apenas se da entre los jóvenes.

Más que con las jergas marginales, las innovaciones juveniles en las variedades coloquiales, se asemejan a los tecnolectos. Un tecnolecto se define habitualmente como la variedad lingüística asociada a una profesión particular. Cuanto más compleja y especializada es la actividad más exige la diferenciación de sus objetos y sus procedimientos y más necesaria es la adopción de denominaciones propias, si bien la lengua general siempre está presente como como fondo y los rasgos, fundamentalmente léxicos, de cada tecnolecto caracterizan sólo a los términos relativos a elementos de la realidad específicamente empleados en la actividad especializada: los nombres de las velas, de los peces, de las enfermedades o de los útiles que se emplean en el robo, por ejemplo. ${ }^{1}$ Por otra parte, los tecnolectos responden al registro

\footnotetext{
1 También habría que considerar propias de tecnolectos a las siglas. Su existencia es sorprendentemente efímera. Del inventario que aporta Seco $(1976,192-193)$ asombra la gran cantidad de las que hoy resultan raras o desconocidas.
} 
en que se utiliza. Es decir, la lengua jurídica o científica, cuyo empleo queda reducido a un registro formal, se acomodará a los rasgos propios de tal registro formal o incluso arcaizante; al contrario, aquellos tecnolectos que se utilizan en registros coloquiales o vulgares, la germanía, por ejemplo, necesariamente han de poseer rasgos de las variedades coloquiales. El uso de los tecnolectos, como de las jergas, desempeña una función crucial en el proceso de inclusión y exclusión de los individuos en los grupos sociales. Fundamentalmente mejoran la comunicación grupal y en este sentido, los tecnolectos funcionan como elementos de cohesión que refuerzan la unidad social. Como señala Martín Rojo (1994: 234-239), permiten crear algo así como un territorio propio del grupo, una realidad en cierta medida alternativa, con sus propios actores, valores y formas de vida. Esto sucede especialmente en las sociedades de redes difusas, en las que existen múltiples contactos lingüísticos y en las que cualquier innovación se difunde rápidamente. En ellas, surge la necesidad de crear vínculos sociales estrechos entre ciertos grupos que sirvan para caracterizarlos como tales y, frente a la importación de innovaciones, se recurre igualmente a la innovación lingüística propia. Por eso, las variedades de los jóvenes sirve como marcador de grupo siempre que reúna dos requisitos: a) tiene que ser aprobada y compartida por los miembros del grupo a cuya coherencia contribuye, dado que las marcas distintivas que no son aceptadas por la generalidad llevan a la extravagancia y al ostracismo y b) ha de incluir elementos que resulten extraños y ajenos a los individuos de mayor edad para excluirlos del grupo (Chambers 1995, 171-176). Así se explica la afinidad por extranjerismos o por términos que pertenecen a las variedades marginales).

Si examinamos con más detalle los rasgos que se atribuyen al sociolecto juvenil del español, tanto en las obras literarias como en descripciones más técnicas, vemos, en primer lugar, que constituyen un inventario muy limitado. El léxico específicamente juvenil se centra en ciertas áreas dominantes. Como cabe identificar una amplia etapa de la juventud con la dedicación escolar e incluso aquellos jóvenes que ya no son estudiantes, hace muy poco tiempo que han dejado de serlo, ello implica que los jóvenes manejan un tecnolecto específico de los estudiantes, con términos como soplar, chuleta, o chuletamen, defi (por deficiente), dire, etc. (Morant Marco 1997). También se han enumerado como formas características de la variedad juvenil de los años ochenta muchos extranjerismos, sobre todo anglicismos referidos a la música o la moda, no generales en español del momento: heavy metal, techno, punk, acid house, mod, soundtrack, bodi, skinhead etc. Tales anglicismos son consecuencia, tal como sucede en el tecnolecto de la física o de la informática, del dominio anglosajón en la creación o en la puesta en circulación de los designata especializados. Otros elementos léxicos son creaciones expresivas de tipo eufemístico o disfemístico que son los que, con cierta frecuencia, proceden de jergas marginales: caballo, 'heroína', mono, 'síndrome de abstinencia', mierda, costo, chocolate, 'hachís', molar, 'gustar', basca, 'grupò de gente', tronco, 'compañero', loro en estar al loro, 'oir la radio', 'estar enterado', bacalo, 'baile rítmico' etc.

Lo que es importante es que esos items léxicos aparecen con finalidad de caracterización grupal y, de la misma manera, desaparecen. Es verdad, no obstante, que sólo en determinados casos y en virtud de la comunicación entre grupos de diferente rango y entidad social, algunas de esas marcas lingüísticas, quizá porque se asocian a realidades nuevas que persisten, tal como sucede con las modas más o menos 
musicales, trascienden a los grupos que las crean y, son éstas las que, difundidas al conjunto de la sociedad, pueden aspirar quizá a perpetuarse.

Si tomamos los datos aportados en las descripciones de los años ochenta, vemos que en unos casos, como corresponde a cualquier innovación, se han producido difusiones a otros ámbitos sociales. Por ejemplo, eso sucede con muchos de los anglicismos arriba citados y también con algunos formantes derivativos introducidos en ese momento: los sufijos -ata de bocata, cubata, ordenata - ota, de pasota, -eras de guaperas o el prefijo super- para el elativo: supercretino, superestupendo (Casado 1981; 1989), formantes que encontramos hoy muy difundidos fuera del ámbito juvenil, incluso en la lengua de la prensa.

Pero, aunque una parte del vocabulario, siguiendo las vías de difusión en la sociedad, se ha generalizado, otra parte, muy relevante, ha desparecido. Del léxico de la movida de los años ochenta, el denominado cheli, recogido por Francisco Umbral (1983), por Ramoncín (1993) o por Rodríguez Díaz (1996), hay numerosos términos que han decaído, especialmente los más caracterizados como marginales: calandrias, tejo, guil, para el dinero, cheira, la navaja, un españa, un cuacua, para ciertas bebidas, cucha 'amante de un preso', guipar, 'ver', hoya, 'cuchara', etc. Otros como bisni o nasti son de excepcional empleo. De cheira, en CORPES XXI aparece solo una sorprendente cita: la cheira de Ockam, sin duda forzada literariamente.

Al ampliar nuestro examen a periodos temporales más extensos, vemos que una cantidad muy alta de los términos de Arniches registrados por Manuel Seco (1970), quizá los más caracterizados afectivamente, son hoy completamente ignorados. Por citar algunos: acatu, 'dinero', achagar, 'azuzar', alicáncano, 'despreciable', menflís, 'tonto', maula, 'holgazán', pipi, 'tonto', rentoy, 'ostentación', repucharse, 'volverse atrás'. En las pioneras obras de Beinhauer (1991 [1930]; 1973) sobre el español coloquial, redactadas originariamente en entorno a los años veinte del siglo pasado, encontramos items léxicos que ahora son desconocidos, no ya por las franjas etarias de jóvenes, sino por el conjunto de los hablantes: fularca, bifurcia, fumuar o tangolio,(con el significado de 'camelo') están ausentes del CREA, del CORPES XXI (Real Academia Española) y del COLA. Suripanta (procedente del texto de una zarzuela, Las juventudes de Telémaco) ha pasado a ser usado solo en obras literarias y no en la variedad coloquial.

A la desaparición le sigue, de manera imparable una renovación del vocabulario, también ámbitos de empleo no muy diferentes. Mancera Rueda y Pano (2013) lo recogen en un medio singularmente favorable a los coloquialismos (López Quero 2003) y que, a su vez, también es usado, sobre todo, por hablantes jóvenes, los chats de internet. En ellos son frecuentes términos como perroflauta, orco, cagamentiras, chismorrón, y nuevos y abundantes anglicismos, fashineta, kisses, etc. Los anglicismos también son numerosos en el corpus COLA que recoge el habla de adolescentes: wok, black, powerline, switch, jackass, entre otros muchos.

\section{EL CAMBIO EN LOS MARCADORES DISCURSIVOS}

Frente a la fácil renovación del léxico, especialmente el especializado, las estructuras gramaticales, si son transparentes y coherentes y si no son afectadas por los cambios 
fónicos, tienden a persistir mucho más. En todo caso, las formas irregulares reflejan una mayor antigüedad, a pesar de que resultan más lábiles y susceptible a cambios debidos a la extensión de las reglas. Pero hay una parcela que está a caballo entre la gramática y el léxico y en la que, además, podemos encontrar algunos de los rasgos de las variedades coloquiales. Es la de los marcadores discursivos, cuyos procesos de creación han sido estudiados muy extensamente (Pons Rodríguez 2010).

Aunque hay muy diversas clasificaciones de marcadores discursivos (Portoles 1998; Martín Zorraquin y Portolés 1999: Duque 2016: 50-52), a efectos prácticos de este trabajo, podemos diferenciar tres grandes grupos de marcadores discursivos. En primer lugar, aquellos marcadores mediante los cuales el emisor organiza la información, la ordena, la aclara, la rectifica o destaca alguno de sus componentes. En este grupo se encuentran los de estructuración y los de foco. Junto a estos marcadores, otros modulan la información tal como el emisor la presenta en relación con la verdad o la probabilidad $y$, en ocasiones, se emplean también para señalar su origen. Por último, citaremos aquellos marcadores que determinan la interacción de los participantes en el coloquio, regulando los turnos, expresando el asentimiento o la corrección con respecto a la información previa (Loureda Lamas y Acín Villa 2010: 24). Y es que los estudios diacrónicos presentan dificultades distintas para cada uno de los grupos, tanto en la documentación de cada tipo, como en las características de su evolución. Así los marcadores pragmáticos de contacto o recepción, que son más frecuentes en la expresión oral, difícilmente se localizan en textos escritos, a la vez que su evolución es menos susceptible de innovaciones, pues entre ellos existen los que cabe considerar como voces naturales. ${ }^{2}$

En relación con los procesos de creación de marcadores discursivos es preciso replantear, como ya han hecho otros autores (Traugott 1995; Estelles Arguedas 2009a: 28-32; Onodera 2011) hasta que punto se ajustan, o no, a los mecanismos propios de la gramaticalización ${ }^{3}$. Ciertamente hay propiedades que se adquieren mediante procesos de gramaticalización que se dan igualmente en los marcadores discursivos. Estos quedan inmovilizados morfológicamente. No sería posible la moción de género o de número en marcadores como bueno o claro, y la moción de tiempo y persona está muy fuertemente restringida en mira, oye (Pons Bordería 1998: 218). También la inmovilidad sintagmática, se produce en gran parte de los marcadores discursivos del español, que suelen ocupar una posición fija, aunque su función puede variar si se altera la posición (Briz y Pons Bordería 2010). Pero los marcadores discursivos son ajenos a lo que se considera un comportamiento específico de los elementos gramaticales, su ligazón (bondedness) con otro elemento léxico (Lehmann 1995: 148). La reducción fónica es otro fenómeno también de escasa importancia en los marcadores discursivos, mientras que el cambio de ámbito estructural (Lehmann 1995: 143-147; Tabor \& Traugott 1998), como señalan Loureda Lamas y Acín Villa (2010: 43-44), se invierte: un marcador discursivo, no solo no reduce su alcance estructural, sino que asume

\footnotetext{
${ }^{2}$ Es el caso, entre otros, de los marcadores de recepción, ah (Vázquez Vega 2003: 172 y ss.) o de contacto, che en español de Valencia y de los países del Plata (García de Diego 1968: 111 y 206).

${ }^{3}$ Tomando en consideración la función de los marcadores, algunos investigadores proponen considerar su habilitación más como pragmatización que como gramaticalización (Diewald 2011: 455-461; Degand y Evers-Vermeul 2015).
} 
funciones que atañen a unidades más extensas. Desde las originarias en el ámbito de una oración, pasan a ejercer funciones discursivas, englobando varias oraciones.

Con todo, el proceso que nos interesa más es el denominado de blanqueo (bleaching), esto es, la eliminación del significado conceptual dirigido a establecer una referencia (Lehmann 1995: 127-131) y, al contrario, la adquisición de una mayor subjetividad, frecuentemente formulada como una mayor implicación del emisor. ¿Tiene lugar este fenómeno en la habilitación de los marcadores discursivos? Company Company (2004a) sostiene que en ellos se produce abiertamente un debilitamiento del significado referencial y ganancia de un significado más abstracto. Efectivamente, este proceso tiene lugar, pero ello no supone la desvinculación total de los marcadores con respecto a los items originarios que poseen significado referencial. Varios investigadores han señalado que los marcadores "en contadas ocasiones se desvinculan del contenido conceptual" (Garcés Gómez 2008: 207; Villar Díaz 2013: 167). Mira no implica un acto directivo de percepción sensible o intelectual, o claro no alude a una determinada gama de intensidad lumínica. Lo que sucede es que se produce una especialización semántica: se aplican algunos de los rasgos semánticos iniciales a la producción discursiva o al discurso en sí para, a partir de tal especialización, dar lugar a determinadas inferencias que guíen la interpretación.

Que en la mayor parte de los marcadores persiste parcialmente su significado léxico aunque desempeñen una función discursiva, y que, además, esta no es enteramente independiente de su significado inicial es perceptible en el caso de los estructuradores discursivos o reformuladores. Por ejemplo, el marcador por fin ratifica el cumplimiento de alguna expectativa; en fin es un reformulador (Garcés Gómez 2014, 93). En los dos se mantienen algunos rasgos semánticos propios de la base léxica, fin, 'extremo' o 'agotamiento de un proceso': en por fin lo, se propone el cumplimiento de expectativas, lo que conlleva precisamente su agotamiento como tales expectativas; en fin remite a la conclusión del proceso argumentativo. Algo similar sucede con los marcadores de modalidad construidos a partir de predicados que califican un componente textual: claro, bueno (Martín Zorraquino 1994a; 1994b). Igualmente es posible mostrar la vinculación que existe en los marcadores de contacto entre interlocutores con sus bases conceptuales. Algunos dan lugar a actos directivos que recaen precisamente sobre el contacto: mira, oye, ¿ Sabes? (Pons Bordería 1998: 215; lliescu 2014); otros, como el italiano prego, son verbos realizativos con una función parecida (Ghezzi, Molinelli 2014). Todos estos marcadores se utilizan configurando actos de habla que implican al interlocutor lo que conlleva, como indican Traugott y Dasher (2001: 19-23), un proceso de intersubjetivización, pero en ellos su significado léxico sigue parcialmente vigente. En suma, en los marcadores discursivos no hay blanqueo, al menos no en el mismo grado que en los procesos de gramaticalización, sino una especialización y ello supone que, en muchos de ellos, las vinculaciones con el paradigma léxico no desaparecen por completo.

Como consecuencia de la vinculación con el léxico descriptivo, los marcadores (si bien no en todas las clases) no terminan de integrarse plenamente en paradigmas cerrados. Para evaluar el grado de clausura de una estructura gramatical existe el recurso de examinar si existe la posibilidad de conmutación entre sus elementos. En los elementos de relación, preposiciones o conjunciones sí existe conmutación, por ejemplo, aunque 
vinol puesto que vino implica una interpretación concesiva frente a la causal. En consecuencia, no pueden aparecer simultáneamente en el mismo nivel dos unidades que se oponen en un paradigma: *aunque, puesto que vino. En algunas clases de marcadores, entre los estructuradores del discurso, por ejemplo, o entre los de ordenación sí cabe establecer igualmente tales oposiciones: en primer lugar / además (Garcés Gómez 2014, 35). Es más dudosa tal oposición entre los marcadores de modalidad, y, desde luego, no existe entre los de contacto: varios marcadores de este grupo pueden emplearse simultáneamente, sin que cambie la función de ninguno de ellos: claro, bueno, oye, mira.

A pesar de lo indicado, con frecuencia las investigaciones sobre marcadores discursivos tienden a incluirlos en paradigmas, unas veces considerándolos constituidos de manera rigurosa (Estellés Arguedas 2009a, 175 y ss.; Pons Bordería 2014), en otros estudios, de manera más flexible (Garcés Gómez 2014, 165). Ello es aceptable si se entienden como estructuras semejantes a los denominados campos léxicos, que admiten ampliaciones o reducciones de sus componentes, pero, en cambio, tales paradigmas son mucho más discutibles si se conciben como los paradigmas gramaticales en los que un cambio afecta sustancialmente a las relaciones existentes en todo el conjunto.

\section{LA HABILITACIÓN RECIENTE DE MARCADORES DISCURSIVOS}

Mientras que los paradigmas gramaticales es difícil que se amplíen con nuevos componentes y, si esto sucede, su proceso de gramaticalización es muy lento, en cambio, una parte importante de los marcadores discursivos, incluso algunos de los pertenecientes a los grupos más estructurados, se han habilitado, según la documentación, muy recientemente, algunos en épocas tan tardías como el siglo XVIII o el XIX. En el inventario de Gregorio Garcés de 1791 (Martín Zorraquino 1998), que no tiene por qué ser exhaustivo, claro está, faltan marcadores como, a lo mejor, desde luego, efectivamente, en resumidas cuentas, vale, allá tú, claro, oye, o sea, por cierto, a propósito, entre otros, todos incluidos en el Diccionario de partículas discursivas del español (Briz, Pons Bordería, Portolés 2008). Ello es una prueba de que muchos de tales marcadores no existían o, en todo caso, que se utilizaban con poca frecuencia. Para algunos marcadores, como por cierto o ciertamente, se les puede reconocer una función argumentativa ya en el siglo XIV (Villar Díaz 2013), pero solo son claramente discursivos en el siglo XVI. Otros, como por lo visto, que se emplea para remitir al origen de la información, solo se consolidan en el siglo XIX (Garcés Gómez 2013, 300-302).

\section{OBSOLESCENCIA}

Como en cualquier investigación diacrónica, sobre gramática y sobre vocabulario, en el estudio de los marcadores discursivos, es preciso estudiar, no solo su creación, sino igualmente su obsolescencia. Es importante, por tanto, examinar marcadores que han estado vigentes durante alguna etapa de la historia del español, pero que han quedado relegados e intentar encontrar explicaciones a su desaparición.

A partir de la documentación que ha publicado A. Ricós Vidal (2008), podemos seguir el proceso de habilitación de un marcador, aosadas, y también su desaparición. Inicialmente se trata de una locución adverbial, compuesta con la preposición a más un 
adjetivo o un participio con función adjetival en -as (osadas). Su sentido, como en muchas locuciones similares, es el de una locución adverbial de modo (idéntico a otras locuciones como a oscuras, a derechas, etc.). ${ }^{4}$ El giro modifica a todo un predicado y significa originalmente 'de manera arriesgada, osada': «Aosadas corred, que por miedo non dexedes nada,/ Fita ayuso e por Guadalfajara» (Cantar de Mio Cid, ed. de Ramón Menéndez Pidal, Madrid, Espasa Calpe, 1964, v. 443). Posteriormente, ya en su función discursiva, aosadas se emplea para calificar como arriesgada, esto es, incierta o insegura una determinada proposición. Funciona, por tanto, como un marcador de modalidad. Sin embargo, a partir de tal suposición, el emisor, solo por hacerla, suscribe un compromiso y con ello enfatiza la aseveración. Implica que el emisor tiene razones suficientes para enunciar la proposición. Es decir, antitéticamente, aosadas pasa a significar, 'con certeza, con seguridad' y es, por tanto, un marcador de refuerzo de la aserción. ${ }^{5}$ Este es el empleo que se documenta a fines del siglo XV en La Celestina: «CELESTINA.-¡Ay como huele toda la ropa en bulléndote! ¡A osadas, que está todo a punto!» (Fernando de Rojas, La Celestina, ed. de Humberto López Morales, Madrid, CUPSA, 1976, p. 122).

Gonzalo de Correas formula en 1627 una descripción muy precisa de su función discursiva: "Aosadas, ke kien lo dixo no mintió. Dízese enkareciendo algo ke kunplidamente se dixo o hizo; kasi lo mesmo ke: «Ahotas»6: konfiadamente. Dicho komo bordonzillo. Algunos: Asuadas`» (Correas 1967: 32 y 603-604).

Aosadas no solo se emplea como marcador tardíamente, sino que, además, tal empleo es efímero. A pesar de la descripción de Correas, los últimos ejemplos que aporta el CORDE son de 1605, de López de Úbeda, Por tanto, no tenemos documentación segura de este marcador más que durante poco más de un siglo. ${ }^{8}$

¿Cómo se puede explicar la evolución de este marcador? Lo primero que cabe observar es que, de acuerdo con lo indicado con respecto a la ausencia de paradigmas de marcadores, la incorporación de nuevos marcadores no conlleva necesariamente la desaparición de otros preexistentes y, a la inversa, la desaparición o decadencia no implica necesariamente su reemplazo por otros funcionalmente equivalentes. En el caso de aosadas, su utilización como marcador no supuso el desuso de otros como sin duda (que), a fe que o seguramente. Todos ellos son utizados ampliamente durante la etapa

\footnotetext{
${ }^{4}$ Nebrija en su Gramática castellana recoge esta locución junto a otras semejantes: «Otros dezimos por rodeo desta preposicion. a. i de algun nombre como apenas aosadas asabiendas adrede.» (Nebrija 1946 [1492], 85).

${ }^{5}$ Podría compararse al actual giro "apuesto a que x" mediante el cual el emisor refuerza la aserción x, a pesar del posible riesgo que está explícito en su formulación.

${ }^{6}$ Ahotas, tal como indica Correas, tiene una suerte y una función semejante a aosadas (Ricós Vidal (2012: 69) aunque se emplea casi exclusiva de la lengua de la comedia pastoril del siglo XVI y de autores que la imitan.

${ }^{7}$ La variante Asuadas que recoge Correas, no solo presenta la fusión de la preposición, sino que hay metátesis que hace más opaca la relación con el étimo.

${ }^{8}$ Amparo Ricós recoge un ejemplo del erudito Bartolomé José Gallardo de 1836: Medio Bruja asmo que es: y aun, a osadas, que si buscarla querrés, cadalnoche la topé (Bartolomé José Gallardo, El Criticón. Número Cuarto, página II, 25. CORDE) Se trata, sin duda, de un uso mediante el cual el autor procura imitar el habla rústica y arcaizante, dado que en el mismo texto aparecen otras formas igualmente desusadas y marginales (cadalnoche, diabro, afrita,etc.). Puede suceder con los marcadores discursivos, al igual que con items léxicos, que caigan en desuso en la lengua general, pero que persistan en variadades dialectales arcaizantes. Ello explica que ahosadas hubiera sido seleccionada por Gallardo como un rasgo arcaizante y también que aosadas siga vivo en el catalán de Valencia.
} 
en que está vigente aosadas. Y, a la inversa, la obsolescencia de esta última forma no se puede vincular a la aparición de algún otro marcador más o menos equivalente.

Es que, al igual que sucede con el léxico, dado que este se configura en series ilimitadas, una nueva incorporación no borra —al menos durante algún tiempo- otros elementos y en consecuencia no es imprescindible la adaptación de todo un conjunto de relaciones funcionales en el interior de un paradigma. $\mathrm{Y}$ si la presión paradigmática no es decisiva en la evolución de los marcadores discursivos, entonces los cambios de cada elemento han de ser considerados como autónomos y, al igual que sucede en el léxico, su evolución semántica depende de otros muy diversoso factores.

En el caso citado citado, como en otros, se hace preciso formular una hipótesis acerca de una utilización tan efímera. En la evolución de los marcadores discursivos existe un factor singular sobre el que es preciso llamar la atención. En ellos, tal como se señala arriba, se mantiene una vinculación semántica con los componentes léxicos de los que proceden. Pues bien, puede suceder que, por la evolución del marcador o por algún cambio en la base orginaria, se vuelva opaca la relación. En tal caso, también queda dificultada la función específica del marcador como guía de inferencias para el destinatario, puesto que su función discursiva deriva (y es una especialización) de ese inicial significado conceptual.

Estos procesos parecen haber tenido lugar con aosadas. Como marcador discursivo tiene un sentido que ha quedado enteramente desligado del de la base sobre la que se formó: osar. El cambio de tipo antitético sobre el que se funda la función como marcador de aosadas lo desliga precisamente del significado de sus componentes iniciales haciéndolo opaco y favoreciendo su desaparición.

\section{CONCLUSIONES}

El artículo ha intentado comprobar si el principio de Spallanzani, la renovación más rápida de los elementos más recientes, se cumple en la lengua. Probablemente se cumple y hay razones para ello. De una parte, en las variedades coloquiales hay una menor presión de la norma externa, al tiempo que las propiedades de la lengua coloquial, proximidad, informalidad, afectividad, dan lugar a circunstancias que facilitan tanto la innovación como el cambio. El principio parece cumplirse también en relación con el léxico coloquial y especialmente en el utilizado por los jóvenes. Su proximidad con los tecnolectos lo hace susceptible de incorporar (muchas veces mediante préstamos) innovaciones. La necesidad de los hablantes de etapas juveniles de individualizarse socialmente frente a otros grupos da lugar a la renovación de las marcas que los caracterizan y, entre esas marcas, se encuentran las lingüísticas.

En otro ámbito, en el de los marcadores discursivos, también se ha documentado, en muchos casos, su habilitación rápida y su fácil obsolescencia. Los cambios semánticos y funcionales que experimentan los marcadores discursivos se acomodan más a los que experimenta el léxico que a los de las unidades gramaticales. La mayor parte de los marcadores discursivos no quedan integrados en paradigmas cerrados $y$, en consecuencia, no desplazan a otras unidades funcionalmente equivalentes. Estas dos características están en consonancia con el hecho de que su habilitación sucede en un 
intervalo de tiempo mucho más reducido que el que se da en los procesos de gramaticalización, puesto que los cambios funcionales que suceden no afectan a todo un conjunto de elementos, ni a las relaciones que se establecen entre ellos, tal como acaece en los cambios gramaticales. Precisamente, cuando los marcadores discursivos se desvinculan de la base léxica inicial, queda dificultada su función y caen en el olvido con mayor rapidez que otros componentes de la gramática.

\section{BIBLIOGRAFÍA}

Bakker, Peter (2000): «Rapid language change: Creolization, intertwinning convergence», en Renfrew, Colin, April McMahon y Larry Trask, eds., Time Depth in Historical Linguistics, Cambridge: The McDonald Institute, 2, pp. 581-620.

Beinhauer, Werner (1991 [1930]): El español coloquial, Madrid: Gredos.

Beinhauer, Werner (1973): El humorismo en el español hablado: (improvisadas creaciones espontáneas), Madrid: Gredos.

Bloomfield, Leonard (1964 [1927]): «Literate and illiterate speech», en D. H. Hymes, ed., Language in culture and society. A reader in linguistics and anthropology, New York: Harper \& Row, pp. 391-396.

Briz Gómez, Antonio (1998): El español coloquial en la conversación, Esbozo de pragmagramática, Barcelona: Ariel.

Briz, Antonio y Pons Bordería, Salvador) (2010): "Unidades, marcadores discursivos y posición», en Loureda Lamas, Óscar y Esperanza Acín Villa, eds., Los estudios sobre marcadores del discurso in español, hoy, Madrid: Arco/Libros, pp. 327-358.

Briz, Antonio., Pons Bordería, Salvador y Portolés, José (coords.) (2008): Diccionario de partículas discursivas del español. En línea, www.dpde.es. Consultado en noviembre de 2018.

Casado Velarde, Manuel (1981): «Un sufijo de la lengua juvenil: -ata», Thesaurus, 36, pp. 323327.

Casado Velarde, Manuel (1989): «Léxico e ideología en la lengua juvenil», en Rodríguez González, Félix, ed., Comunicación y lenguaje juvenil, Madrid: Editorial Fundamentos, pp. 167-178.

Chambers, J. K. (1995): Sociolinguistic Theory. Linguistic Variation and its Social significance, Oxford UK \& Cambridge USA: Blackwell.

COLA. Corpus oral de lenguaje adolescente. http://colam.org/publikasjoner/corpuslenguaj eadoles.htm. Consulta diciembre de 2018.

Company Company, Concepción (2004a):.«¿Gramaticalización o desgramticalización? Reanálisis y subjetivización de verbos como marcadores discursivos en la historia del español», RFE, 84, pp. 29-66.

Company Company, Concepción (2004b): «Gramaticalización por subjetivización como prescindibilidad de la sintaxis», NRFH, 52, pp. 1-27.

Correas G. (1967): Vocabulario de refranes y frases proverbiales. Texte établi...par Louis Combet, Bordeaux: Féret et fils.

Degand, Liesbeth y Jacqueline Evers-Vermeul (2015): «Grammaticalization or pragmaticalization of discourse markers?», Journal of Historical Pragmatics, 16, 1, pp. 5985.

Diewald, Gabrielle (2011): «Grammaticalization and pragmaticalization», en Narrog, Heiko y Bernd Heine, eds., The Oxford Handbook of Grammaticalization, Oxford: Oxford University Press, pp. 450-461.

Duque, Eladio (2016): Las relaciones de discurso, Madrid: Arco/Libros.

Eckert, Penelope (1989): Jocks and Burnouts: Social Categories and Identidy in the High School, New York/Londres: Teachers College Press.

Estellés Arguedas, María (2009a): Gramaticalización y gramaticalizaciones. El caso de los marcadores del discurso de digresión en español. Tesis doctoral. Valencia: Universitat de València. Obtenida en http://roderic.uv.es/handle/10550/15767 [Consulta: 01/2019].

Estellés Arguedas, María (2009b): «Un caso atípico de gramaticalización (I): el valor epistémico del marcador por cierto», RILCE, 25, 2, pp. 319-339.

Garcés, Gregorio (1852, 11791): Fundamento del valor y elegancia de la lengua castellana, Madrid: Rivadeneira.

Garcés Gómez, Pilar (2008): La organización del discurso: marcadores de ordenación y de reformulación, Madrid: Iberoamericana/Vervuert.

Garcés Gómez, Pilar (2013): «La formación y evolución del paradigma de los operadores discursivos matizadores de la verdad del enunciado», en Pilar Garcés Gómez, ed., Los adverbios con función discursiva: procesos 
de formación y evolución, Madrid: Iberoamericana/Vervuert, pp. 275-316.

Garcés Gómez, Pilar (2014): Diacronía de los marcadores discursivos y representación in un diccionario histórico, La Coruña: Universidade da Coruña.

García de Diego, Vicente (1968): Diccionario de voces naturales, Madrid: Aguilar.

Ghezzi, Chiara y Molinelli, Piera (2014): «Deverbal pragmatic markers from Latin to Italian (Lat. QUAESO and It. prego): The cyclic nature of functional developments)», en Ghezzi, Chiara y Piera Molinelli, eds., Discourse and Pragmatic Markers from Latin to the Romance Languages, Oxford: Oxford University Press, pp. 61-85.

Havranek, Bohuslav (1966 [1936]): «Zum Problem der Norm in der heutigen Sprachwissenschaft und Sprachkultur»,. en Vachek, Josef, ed., A Prague School Reader in Linguistics, Bloomington \& London: Indiana University Press, pp. , 413-420.

Holloway, Charles E. (1997): Dialect Death. The case of Brule Spanish, Amsterdam, Benjamins.

Iliescu, Maria (2014): "Call markers in French, Italian, and Romanian», en Ghezzi, Chiara y Piera Molinelli, eds., Discourse and Pragmatic Markers from Latin to the Romance Languages, Oxford: Oxford University Press, pp. 29-40.

Lázaro Carreter, Fernando (1980): «Cambio lingüístico y generaciones», Estudios de Lingüística, Barcelona: Crítica, pp. 11-26.

Lehmann, Christian (1995): Thoughts on Grammaticalization, München: LINCOM Europa.

López Quero, Salvador (2003): El lenguaje de los "chats», Granada: Port Royal Ediciones.

Loureda Lamas, Óscar y Acín Viña, Esperanza, eds. (2010): Los estudios sobre marcadores del discurso en español, hoy, Madrid: Arco/Libros.

Mancera Rueda, Ana y Pano, Ana (2013): El español coloquial en las redes sociales, Madrid: Arco/Libros.

Martín Rojo, Luisa, (1994): «The jargon of delinquents and the study of conversacional dynamics», Journal of Pragmatics, 21, pp. 243-289.

Martin Zorraquino, M. Antonia (1994a): «Algunas observaciones sobre claro como operador pragmatico en español actual», en Gerold Hilty, ed., Actes du XXe . Congres International de Linguistique et Philologie Romanes, Tübingen: Francke, vol. 1, pp. 467-78.

Martin Zorraquino, M. Antonia (1994b): «Bueno como operador pragmatico en español actual», . en Alegría Alonso, et al., eds., II
Encuentro de linguistas y filologos de Espana y Mexico, Salamanca: Universidad de Salamanca, pp. 402-412.

Martin Zorraquino, M. Antonia (1998): «Dos notas a proposito del Tratado de las particulas de $\mathrm{G}$. Garces (1791). (Un precedente en el estudio de los marcadores del discurso)», en Ramon Trives, Estanislao y H. Provencio Garrigos, eds., Estudios de lingüística textual. Homenaje al Profesor Muñoz Cortes, Murcia, Universidad de Murcia, pp. 307-315.

Martin Zorraquino, M. Antonia (2003): «Marcadores del discurso y diccionario: sobre el tratamiento lexicografico de desde luego", en M. Teresa Echenique Elizondo y Juan Sanchez Mendez (coords.). Homenaje al Prof. Dr. Gunther Haensch, Madrid: Gredos, pp. 439-452.

Martín Zorraquino, M. Antonia y José Portolés (1999): Los marcadores del discurso, en Bosque, Ignacio y Violeta Demonte, eds., Gramática descriptiva de la lengua española, Madrid: Espasa Calpe, vol. III, pp. 4051-4213.

Morant Marco, Ricardo (1997): «El lenguaje de los estudiantes», II Jornadas de Lingüística, Cádiz: Universidad de Cádiz, 91-104.

Nebrija, Antonio (1946 [1492]): Gramática Castellana. Edición crítica de Pascual Galindo Romeo y Luis Ortiz Muñoz, Madrid: Edición de la Junta del Centenario.

Onodera, Noriko O. (2011): «The Grammaticalization of Discourse Markers», en Heiko Narrog y Bernd Heine, eds., The Oxford Handbook of Grammaticalization, Oxford: Oxford University Press, pp. 614-624.

Pons Bordería, Salvador (1998): «Oye y mira o los límites de la conexión», en. Martín Zorraquino, M. Antonia y Estrella Montolío Durán, eds., Marcadores del discurso, Teoría y análisis, Madrid: Arco/Libros, pp. 213-228.

Pons Bordería, S Salvador (2014): «Paths of grammaticalization in Spanish o sea», en Ghezzi, Chiara y Piera Molinelli, eds., Discourse and Pragmatic Markers from Latin to the Romance Languages, Oxford: Oxford University Press, pp. 109-136.

Pons Rodríguez, Lola (2010): «Los marcadores del discurso en la historia del español», en Loureda Lamas, Óscar y Esperanza Acín Villa, eds., Los estudios sobre marcadores del discurso en español, hoy, Madrid: Arco/Libros, pp. 523-616.

Portolés, José (1998): Marcadores discusivos, Barcelona: Ariel.

Ramoncín [José Ramón Julio Márquez Martínez] (1993): El tocho cheli. Diccionario de jergas, Madrid: Ediciones Temas de Hoy.

Real Academia Española, Corpus diacrónico del español (CORDE). Banco de datos en línea: 
http.//www.rae.es. Consultas en diciembre de 2018.

Real Academia Española, Corpus de Referencia del Español Actual (CREA). Banco de datos en línea: http.//www.rae.es. Consultas en diciembre de 2018.

Real Academia Española, Corpus del español del siglo XXI (CORPES XXI). Banco de datos en línea: http.//www.rae.es. Consultas en diciembre de 2018.

Ricós Vidal, Amaparo (2008): «Locuciones adverbiales con preposición a y adjetivo in el español y el portugués del siglo XVI. Estudio histórico», en Actas del VII Congreso Internacional de la Lengua Española. Mérida (Yucatán). 4-8 de septiembre de 2006, Madrid: Arco/Libros, pp.1035-1054.

Ricós Vidal, Amparo (2012): A reculas, a hotas, a sabiendas, a la deshilada, a la callada, a la sorda: más sobre locuciones adverbiales, en García Valle, Adela et al., eds., Fablar bien e tan mesurado: veinticinco años de investigación diacrónica in Valencia: estudios ofrecidos a M. Teresa Echenique Elizondo in conmemoración de su cátedra, Valencia: Tirant Humanidades, pp. 68-86.

Rivière, Margarita (1989): «Moda de los jóvenes: un lenguaje adulterado», en Rodríguez González, Félix, ed., Comunicación y lenguaje juvenil, Madrid: Editorial Fundamentos, pp. 71-77.

Rodríguez Díez, Bonifacio (1996): «Argot y lenguaje coloquial» en Briz, Antonio et al., eds., Pragmática y gramática del español hablado, El español coloquial, Zaragoza: Pórtico, pp. 225-239.

Sanmartín, Julia (2006, [1998]): Diccionario de argot. Nueva versión, Madrid: Espasa Calpe.

Sapir, Edward (1958): "Time perspective in Aboriginal American Culture: A study in
Method», en: Selected Writings of Edward Sapir in Language, Culture and personality, ed., David G. Mandelbaum, Berkeley: University of California., pp. 389-462.

Seco, Manuel (1970): Arniches y el habla de Madrid, Madrid: Alfaguara.

Seco, Manuel (1977): El léxico de hoy, en: Rafael Lapesa (coord.), Comunicación y lenguaje, Madrid: Karpos, pp. 183-201.

Spallanzani, Lorenzo (1768): Prodromo di un'opera da imprimersi sopra le riproduzioni animali, Modena: Montanari.

Tabor, Whitney y Elisabeth Closs Traugott (1998): «Structural scope expansion and grammaticalization», en Giancalone Ramat, Anna y Paul J. Hopper, eds., The Limits of Grammaticalization,

Amsterdam/Philadelphia: John Benjamins, pp.229-272.

Traugott, Elisabeth Closs (1995): The role of grammaticalization in the development of discourse markers. Paper presented in ICHL XII, Manchester. Disponible en: http://www.stanford.edu/ traugott/papers/dis course.pdf.

Traugott, Elisabeth Closs y Richard B. Dasher. (2001): Regularity in Semantic Change, Cambridge: Cambridge University Press.

Umbral, Francisco (1983): Diccionario cheli, Barcelona: Grijalbo.

Vázquez Vega, Nancy (2003): Marcadores discusivos de recepción, Santiago de Compostela: Universidad de Santiago de Compostela.

Villar Díaz, María Belén (2013) «La evolución de los adverbios y locuciones adverbiales de modalidad epistémica», en Pilar Garcés Gómez, Los adverbios con función discursiva: procesos de formación y evolución, Madrid: Iberoamericana/Vervuert, pp. 157-199. 